\title{
SHARP DISTORTION THEOREMS FOR QUASICONFORMAL MAPPINGS
}

\author{
G. D. ANDERSON, M. K. VAMANAMURTHY, AND M. VUORINEN*
}

\begin{abstract}
Continuing their earlier work on distortion theory, the authors prove some dimension-free distortion theorems for $K$-quasiconformal mappings in $R^{n}$. For example, one of the present results is the following sharp variant of the Schwarz lemma: If $f$ is a $K$-quasiconformal self-mapping of the unit ball $B^{n}, n \geqslant 2$, with $f(0)=0$, then $4^{1-K^{2}}|x|^{K} \leqslant|f(x)| \leqslant 4^{1-1 / K^{2}}|x|^{1 / K}$ for all $x$ in $B^{n}$.
\end{abstract}

1. Introduction. Some fundamental distortion properties of $K$-quasiconformal mappings of the unit ball $B^{n}$ in $R^{n}$ are expressed by the two facts that these mappings (1) are Hölder continuous and (2) satisfy a generalized Schwarz lemma. Such results were first obtained in the plane case by L. V. Ahlfors [Ah1] and J. Hersch and A. Pfluger [HP]. Later the multidimensional case was studied by E. D. Callender [C], F. W. Gehring [G], Yu. G. Reshetnyak [R1, R2], B. V. Shabat [Sh], and O. Martio, S. Rickman, and J. Väisälä [MRV]. These $n$-dimensional results depend essentially on $n$ and they contain constants that are unbounded as $n$ tends to $\infty$. The present authors [AVV] recently improved these results by showing that there also exists a dimension-free distortion theory of $K$-quasiconformal mappings in $R^{n}$.

In the present paper we continue our earlier work and examine two conjectures due to A. V. Sychev and to T. Iwaniec, respectively. After this we prove a dimension-free variant of a distortion theorem due to F. W. Gehring and B. G. Osgood [GO, Theorem 3].

Before stating the main results of this paper we introduce some necessary notation and recall those well-known results which will be used throughout. The Grötzsch and Teichmüller condensers in $R^{n}$ are denoted by $R_{G, n}(s), s>1$, and $R_{T, n}(t)$, $t>0$, respectively. Their (conformal) capacities are denoted as in [AVV] by

$$
\begin{aligned}
& \gamma(s)=\gamma_{n}(s)=\operatorname{cap} R_{G, n}(s), \quad s>1, \\
& \tau(t)=\tau_{n}(t)=\operatorname{cap} R_{T, n}(t), \quad t>0 .
\end{aligned}
$$

These functions satisfy the basic functional identity

$$
\gamma(s)=2^{n-1} \tau\left(s^{2}-1\right), \quad s>1 .
$$

We sometimes omit the subscript $n$ if there is no danger of confusion.

Received by the editors October 20,1986. Presented by the second author to the New Zealand Mathematical Society at its May 1987 annual meeting in Hamilton.

1980 Mathematics Subject Classification (1985 Revision). Primary 30C60; Secondary 30C75.

* Research supported in part by an exchange award between the Academy of Finland and the U. S. National Science Foundation. 
Next, for $K>0$ and $n \geqslant 2$, we define a homeomorphism $\varphi_{K}=\varphi_{K, n}:[0,1] \rightarrow[0,1]$ by $\varphi_{K}(0)=0, \varphi_{K}(1)=1$ and

$$
\varphi_{K}(r)=\varphi_{K, n}(r)=\frac{1}{\gamma_{n}^{-1}\left(K \gamma_{n}(1 / r)\right)}
$$

when $0<r<1$. For $K \geqslant 1$ and $n \geqslant 2$, we also set

$$
\varphi_{K}^{*}(r)=\varphi_{K, n}^{*}(r)=\sup \left\{|f(x)|: f \in Q C_{K}\left(B^{n}\right), f(0)=0,|x|=r\right\}
$$

$r \in[0,1)$, and $\varphi_{K, n}^{*}(1)=1$, where $Q C_{K}\left(B^{n}\right)$ is the set of all $K$-quasiconformal mappings $f$ of $B^{n}$ into $B^{n}$. By the quasiconformal Schwarz lemma [Sh; MRV, 3.1; W] we obtain

$$
\varphi_{K, n}^{*}(r) \leqslant \varphi_{K, n}(r)
$$

for all $K \geqslant 1, n \geqslant 2$, and $r \in[0,1]$. By [LV, p. 64, Theorem 3.1],

$$
\varphi_{K, 2}^{*}(r)=\varphi_{K, 2}(r)
$$

for all $K \geqslant 1$ and $r \in[0,1]$. Whether the $n$-dimensional analogue of (1.5) holds for $n \geqslant 3$ is an open problem. By [W; AVV, 4.10, 4.14], for all $n \geqslant 2$ and $K \geqslant 1$,

$$
r^{\alpha} \leqslant \varphi_{K, n}(r) \leqslant \lambda_{n}^{1-\alpha} r^{\alpha} \leqslant 2^{1-1 / K} K r^{\alpha}, \quad \alpha=K^{1 /(1-n)},
$$

where $\lambda_{n} \in\left[4,2 e^{n-1}\right)$ [An, (4)] is the Grötzsch ring constant depending only on $n$. The weaker bound $\varphi_{K, n}(r) \leqslant \lambda_{n} r^{\alpha}$ is given in [MRV, 2.8].

It was conjectured by A. V. Sychev [Sy, p. 89, Remark 2] that

$$
\varphi_{K, n}^{*}(r) \leqslant 4^{1-\alpha} r^{\alpha}, \quad \alpha=K^{1 /(1-n)}
$$

for all $n \geqslant 3$ and $K \geqslant 1$. Since $\lambda_{2}=4$ [LV, p. 61], inequality (1.7) follows from (1.6) [W] when $n=2$. But since $\lim _{n} \lambda_{n}=\infty$ [An], one cannot derive (1.7) from (1.6) and (1.4) for general $n$. Furthermore, it follows from [AVV], as we shall point out below in Remark 2.28, that (1.7) with $\varphi_{K, n}^{*}$ replaced by $\varphi_{K, n}$ is false. However, by using Lemma 2.3 below (an improved version of [Vu2, 3.5]) along with (1.6) and (1.4) we are able to prove our first theorem, which is very close to Sychev's conjecture.

1.8. TheOREM. For $n \geqslant 2, K \geqslant 1$, and $r \in[0,1]$ the following inequality holds:

$$
\varphi_{K, n}^{*}(r) \leqslant 4^{1-1 / K^{2}} r^{1 / K} .
$$

This inequality is sharp when $K=1$. Furthermore, the constant 4 cannot be replaced by a smaller one independent of $K$.

Our second theorem gives an affirmative answer to a conjecture communicated by T. Iwaniec to M. Vuorinen during the 1978 ICM in Helsinki. The theorem is applicable to a class of mappings that is larger than $Q C_{K}\left(B^{n}\right)$, namely the class $Q R_{K}\left(B^{n}\right)$ of all $K$-quasiregular mappings of $B^{n}$ into $B^{n}$. In a less precise form our theorem is contained in [R1, R2; R3, p. 38; MRV, 3.1]. For the definition and some basic properties of quasiregular mappings the reader is referred to [MRV, I, R3]. 
1.10. ThEOREM. For $n \geqslant 2, r \in(0,1)$, and $K \in[1, \infty)$ there exists a number $a(r)$ with $\lim _{r \rightarrow 0} a(r)=1$ such that if $f \in Q R_{K}\left(B^{n}\right)$ then

$$
|f(x)-f(y)| \leqslant a(r) \lambda_{n}^{1-\alpha}|x-y|^{\alpha} \leqslant a(r) 2^{1-1 / K} K|x-y|^{\alpha}
$$

for all $x, y \in \bar{B}^{n}(r)$, where $\alpha=K^{1 /(1-n)}$.

We shall actually prove a sharp version of Theorem 1.10, which is perhaps new even for $n=2$; the above simplified formulation sacrifices the sharpness.

For our third result we require the quasihyperbolic metric $k_{D}$ of a proper subdomain $D$ of $R^{n}$. For $a, b \in D$ we set

$$
k_{D}(a, b)=\inf _{\gamma} \int_{\gamma} d(x, \partial D)^{-1} d s
$$

[GP, GO], where the infimum is taken over all rectifiable arcs $\gamma$ joining $a$ and $b$ in $D$. We shall prove the following dimension-free version of a distortion theorem due to Gehring and Osgood [GO, Theorem 3].

1.12. TheOREM. Let $f: D \rightarrow D^{\prime}$ be a $K$-quasiconformal mapping, where $D$ and $D^{\prime}$ are proper subdomains of $R^{n}$. Then

$$
\begin{aligned}
k_{D^{\prime}}(f(x), f(y)) & \leqslant c(K) \max \left\{k_{D}(x, y)^{\alpha}, k_{D}(x, y)\right\} \\
& \leqslant c(K) \max \left\{k_{D}(x, y)^{1 / K}, \ell_{D}(x, y)\right\}
\end{aligned}
$$

for all $x, y \in D$, where $\alpha=K^{1 /(1-n)}$ and $c(K)$ is a constant depending only on $K$.

The proof of this theorem makes use of recent results on Teichmüller's modulus problem in $R^{n}$ [Vu3]. We also obtain lower and upper bounds for the least constant $c(K)$ for which this theorem holds.

2. The extremal distortion function $\varphi_{K, n}^{*}(r)$. We shall adopt the relatively standard notation and terminology of $[\mathbf{V}]$. Unit vectors in the directions of the rectangular coordinate axes in $R^{n}$ are denoted by $e_{1}, \ldots, e_{n}$. For $x \in R^{n}$ and $r>0$ we let $B^{n}(x, r)=\left\{z \in R^{n}: \quad|x-z|<r\right\}, \quad S^{n-1}(x, r)=\partial B^{n}(x, r), \quad B^{n}(r)=B^{n}(0, r)$, $S^{n-1}(r)=\partial B^{n}(r), B^{n}=B^{n}(1)$, and $S^{n-1}=\partial B^{n}$.

The set of Möbius transformations in $\bar{R}^{n}=R^{n} \cup\{\infty\}$ is denoted by $G M\left(\bar{R}^{n}\right)$, and the subset of sense-preserving transformations by $M\left(\bar{R}^{n}\right)$. For nonempty $D \subset \bar{R}^{n}$ we let $G M(D)=\left\{f \in G M\left(\bar{R}^{n}\right): f D=D\right\}$ and $M(D)=\left\{f \in M\left(\bar{R}^{n}\right)\right.$ : $f D=D\}$. We shall require the Poincaré metric $\rho(x, y)$ on $B^{n}$ defined by

$$
\tanh ^{2} \frac{\rho(x, y)}{2}=\frac{|x-y|^{2}}{|x-y|^{2}+\left(1-|x|^{2}\right)\left(1-|y|^{2}\right)}
$$

for $x, y \in B^{n}$ or, equivalently, by

$$
\sinh ^{2} \frac{\rho(x, y)}{2}=\frac{|x-y|^{2}}{\left(1-|x|^{2}\right)\left(1-|y|^{2}\right)}
$$


for $x, y \in B^{n}$ (cf. [B, p. 40]). In particular,

$$
\rho(0, x)=\log \frac{1+|x|}{1-|x|}=2 \operatorname{arctanh}|x|
$$

for $x \in B^{n}$. It is a basic fact that $\rho$ is a $G M\left(B^{n}\right)$-invariant function-that is, $\rho(x, y)=\rho(T x, T y)$ for all $T \in G M\left(B^{n}\right)$ and all $x, y \in B^{n}$.

The results in this section rely essentially on the following lemma, which is a sharpened version of [ $\mathbf{V u 2}, 3.5]$. By means of (1.1) one can show that this lemma is equivalent to the Schwarz lemma (1.4) if $n=2$, while for $n \geqslant 3$ a different result is obtained (see (2.15) below).

2.3. Lemma. Let $K \geqslant 1, n \geqslant 2$, and $f \in Q C_{K}\left(B^{n}\right)$. Then

$$
\sinh ^{2} \frac{\rho^{\prime}}{2} \leqslant \tau^{-1}\left(\frac{1}{K} \tau\left(\sinh ^{2} \frac{\rho}{2}\right)\right)
$$

and

$$
\tanh \frac{\rho^{\prime}}{4} \leqslant 2\left(\tanh \frac{\rho}{4}\right)^{1 / K}
$$

where $\rho=\rho(x, y)$ and $\rho^{\prime}=\rho(f(x), f(y))$ for all $x, y \in B^{n}$.

Proof. The conformal invariant $\lambda_{B^{n}}(x, y)$ examined in [Vu2] has the following explicit expression (see [ $\mathbf{V u 2}, 2.23])$ :

$$
\lambda_{B^{n}}(x, y)=\frac{1}{2} \tau_{n}\left(\sinh ^{2} \frac{\rho(x, y)}{2}\right) .
$$

The proof of (2.4) now follows directly from (2.6) and [Vu2, Lemma 3.1(2), 2.20]. It follows from (2.6) and [Vu2, 2.14(2)] that

$$
\lambda_{B^{n}}(x, y) \geqslant c_{n} \log (\operatorname{coth} \rho(x, y) / 4),
$$

while

$$
\lambda_{f B^{n}}(f(x), f(y)) \leqslant \lambda_{B^{n}}(f(x), f(y)) \leqslant \frac{c_{n}}{2} \log \left(4 \operatorname{coth}^{2} \frac{\rho(f(x), f(y))}{4}\right)
$$

by (2.6) and [ $\mathbf{V u 2},(5.23)]$. Here $c_{n}$ is a constant depending only on $n[\mathbf{V}, 10.9]$. These two inequalities together with [ $\mathbf{V u 2}, 3.1(2)]$ yield inequality (2.5).

2.7. THEOREM. If $f \in Q C_{K}\left(B^{n}\right)$ and $f(0)=0$, then

$$
|f(x)| /\left(1+\sqrt{1-|f(x)|^{2}}\right) \leqslant 2\left(|x| /\left(1+\sqrt{1-|x|^{2}}\right)\right)^{1 / K}
$$

for all $x \in B^{n}$.

Proof. The proof follows immediately from (2.5) and the observation that

$$
\tanh \left(\frac{1}{4} \log \frac{1+s}{1-s}\right)=\frac{s}{1+\sqrt{1-s^{2}}}
$$

for $s \in[0,1)$. 
2.8. Corollary. For all $n \geqslant 2, K \geqslant 1$, and $r \in[0,1]$,

$$
\varphi_{K, n}^{*}(r) \leqslant \min \{2, K\} 2^{1-1 / K} r^{1 / K} .
$$

Proof. Let $f \in Q C_{K}\left(B^{n}\right)$ with $f(0)=0$, and $|x|=r<1$. Since there is nothing to prove if $|f(x)| \leqslant|x|=r$, we assume that $|f(x)|>|x|$. Then by Theorem 2.7

$$
\begin{aligned}
|f(x)| & \leqslant 2 \frac{1+\left(1-|f(x)|^{2}\right)^{1 / 2}}{\left(1+\left(1-|x|^{2}\right)^{1 / 2}\right)^{1 / K}}|x|^{1 / K} \leqslant 2\left(1+\left(1-|x|^{2}\right)^{1 / 2}\right)^{1-1 / K}|x|^{1 / K} \\
& \leqslant 2 \cdot 2^{1-1 / K}|x|^{1 / K} .
\end{aligned}
$$

From definition (1.3) it now follows that $\varphi_{K, n}^{*}(r) \leqslant 2^{2-1 / K} r^{1 / K}$. Finally, since $\varphi_{K, n}(r) \leqslant K \cdot 2^{1-1 / K} r^{1 / K}$ and $\varphi_{K, n}^{*}(r) \leqslant \varphi_{K, n}(r)$ by (1.6) and (1.4), we obtain (2.9).

2.10. Corollary. For $n \geqslant 2, K \geqslant 1$, and $r \in[0,1]$, the extremal distortion function $\varphi_{K, n}^{*}$ satisfies

$$
\varphi_{K, n}^{*}(r) \leqslant 4^{1-1 / K^{2}} r^{1 / K}
$$

and

$$
\varphi_{K, n}^{*}(r) \leqslant 8^{1-1 / K} r^{1 / K} .
$$

Furthermore, the constant 4 in (2.11) cannot be replaced by a smaller absolute constant.

Proof. One can derive both inequalities from (2.9) by elementary methods, considering separately the two cases $1 \leqslant K \leqslant 2$ and $K \geqslant 2$. We shall give details only for (2.11).

If $1 \leqslant K \leqslant 2$, then by (2.9) it suffices to prove that $4^{1-1 / K^{2}} \geqslant 2^{1-1 / K} K$ or, equivalently, that

$$
2^{1-2 / K^{2}+1 / K} \geqslant K
$$

Let $g(K)=\left(1-2 K^{-2}+K^{-1}\right) \log 2-\log K$. Then $g(1)=g(2)=0$ and $g^{\prime \prime}(K)<0$ for $1 \leqslant K \leqslant 2$; (2.13) holds accordingly.

In case $K \geqslant 2,(2.11)$ follows from (2.9) and the easily verified fact that $4^{1-1 / K^{2}} \geqslant$ $2^{1-1 / K} \cdot 2$ for such $K$.

To prove that 4 is the best constant in (2.11) we consider the case $n=2$. Suppose that $\varphi_{K, 2}(r) \leqslant d^{1-1 / K^{2}} r^{1 / K}$ for all $K \geqslant 1$ and all $r \in[0,1]$. Then by [LV, p. 65]

$$
4^{1-1 / K}=\lim _{r \rightarrow 0} r^{-1 / K} \varphi_{K, 2}(r) \leqslant d^{1-1 / K^{2}} .
$$

Letting $K \rightarrow \infty$ yields $d \geqslant 4$ as desired.

The proof of Theorem 1.8 follows from $(2.11)$ and the fact that $\varphi_{1, n}^{*}(r)=r$. It should be observed that for $n \geqslant 3$ and $K>1$, the exponent $1 / K$ in (2.11) is not equal to the best exponent $\alpha=K^{1 /(1-n)}$ (see (1.4) and (1.6)). On the other hand, the upper bounds in Corollary 2.10 are bounded as $K$ tends to $\infty$, while the constant $2^{1-1 / K} K$ in (1.6) fails to have this property. 
2.14. Remark. For a function $f \in Q C_{K}\left(B^{n}\right)$ with $f(0)=0$ one obtains from (2.11), (1.4), and (1.6) the following improved variant of the Schwarz lemma.

$$
|f(x)| \leqslant \min \left\{2^{1-1 / K} K|x|^{\alpha}, 4^{1-1 / K^{2}}|x|^{1 / K}\right\}
$$

for all $x \in B^{n}$. By exploiting inequality (2.15) one can slightly improve the functions $\eta$ and $\Theta$ constructed in [AVV, (5.22) and AVV, Theorem 6.2], respectively.

2.16. Definition. For $n \geqslant 2, K \geqslant 1$, we set

$$
\varphi_{1 / K, n}^{*}(r)=\inf \left\{|f(x)|: f \in Q C_{K}\left(B^{n}\right), f(0)=0,|x|=r, f B^{n}=B^{n}\right\}
$$

for $r \in[0,1)$ and $\varphi_{1 / K, n}^{*}(1)=1$, extending definition (1.3).

2.18. REMARK. If $K \geqslant 1$ and $n \geqslant 2$ then, by (1.4) and the proof of [AVV, Theorem 4.10],

$$
\varphi_{1 / K, n}(r) \leqslant \varphi_{1 / K, n}^{*}(r) \leqslant r^{\beta} \leqslant r^{\alpha} \leqslant \varphi_{K, n}^{*}(r) \leqslant \varphi_{K, n}(r),
$$

where $\alpha=K^{1 /(1-n)}=1 / \beta$. Moreover, by the proof of [AVV, Theorem 4.9],

$$
\varphi_{1 / K, n}^{*}(r) \leqslant \varphi_{\alpha, 2}(r) \leqslant \varphi_{\beta, 2}(r) \leqslant \varphi_{K, n}^{*}(r)
$$

(cf. also (1.5)).

2.19. ReMARK. It is known that $\varphi_{K, 2}^{*}=\varphi_{K, 2}$ for $K \geqslant 1$ (cf. (1.5)). One may also show that $\varphi_{1 / K, 2}^{*}=\varphi_{1 / K, 2}$ for $K \geqslant 1$ by considering the inverse of the Grötzsch extremal $K$-quasiconformal mapping of $B^{2}$ onto itself (cf. [LV, Theorem 3.1, p. 64]).

2.20. LemMA. For $n \geqslant 2, K \geqslant 1$, and $r \in[0,1]$,

$$
\varphi_{1 / K, n}^{*}(r) \geqslant 2^{1-K} K^{-K} r^{\beta}
$$

where $\beta=K^{1 /(n-1)}=1 / \alpha$.

Proof. By [AVV, Theorem 4.2 and (4.12)] we see that $\varphi_{1 / K, n}^{*}(r) \geqslant \varphi_{1 / K, n}(r) \geqslant$ $\lambda_{n}^{1-\beta} r^{\beta}$. But from the proof of [AVV, Corollary 4.14] and the fact that $1-\alpha=$ $(\beta-1) \alpha$ it follows that $\lambda_{n}^{\beta-1} \leqslant 2^{(1-1 / K) / \alpha} K^{1 / \alpha} \leqslant 2^{K(1-1 / K)} K^{K}=2^{K-1} K^{K}$. Thus $\lambda_{n}^{1-\beta} \geqslant 2^{1-K} K^{-K}$.

2.21. THEOREM. Let $f$ be a $K$-quasiconformal mapping of $B^{n}$ onto $B^{n}$ with $f(0)=0$. Then $|f(x)| \geqslant 2^{1-2 K}|x|^{K}$ for all $x \in B^{n}$.

Proof. First, if $|f(x)| \geqslant|x|$, there is nothing to prove. Next, suppose $|f(x)|<|x|$. Since $f B^{n}=B^{n}$, Theorem 2.7 applied to $f^{-1}$ yields

$$
|x| /\left(1+\sqrt{1-|x|^{2}}\right) \leqslant 2\left(|f(x)| /\left(1+\sqrt{1-|f(x)|^{2}}\right)\right)^{1 / K} .
$$

Solving this inequality for $|f(x)|$ and making some obvious estimates gives

$$
\begin{aligned}
|f(x)| & \geqslant \frac{1+\sqrt{1-|f(x)|^{2}}}{\left(1+\sqrt{1-|x|^{2}}\right)^{K}}\left(\frac{|x|}{2}\right)^{K} \\
& \geqslant\left(1+\sqrt{1-|x|^{2}}\right)^{1-K}\left(\frac{|x|}{2}\right)^{K} \geqslant 2^{1-2 K}|x|^{K},
\end{aligned}
$$

as desired. 
2.22. Corollary. For $n \geqslant 2, K \geqslant 1$, and $r \in[0,1]$,

$$
\varphi_{1 / K, n}^{*}(r) \geqslant \max \left\{2^{1-K} K^{-K_{r} \beta}, 2^{1-2 K_{r} K}\right\} .
$$

Proof. This is clear by Lemma 2.20 and Theorem 2.21 .

2.23. Corollary. For $n \geqslant 2, K \geqslant 1$, and $r \in[0,1]$,

$$
\varphi_{1 / K, n}^{*}(r) \geqslant 4^{1-K^{2}} r^{\beta} \text {. }
$$

Proof. By Corollary 2.22 it suffices to show that

$$
2^{1-K} K^{-K} \geqslant 4^{1-K^{2}} \text { for } K \geqslant 1 .
$$

It is easy to see that this is equivalent to

$$
h(K) \equiv K \log K+\left(1+K-2 K^{2}\right) \log 2 \leqslant 0
$$

for $K \geqslant 1$. But since $h(1)=0, h^{\prime}(1)=1-3 \log 2<0$, and $h^{\prime \prime}(K)=1 / K-$ $4 \log 2 \leqslant 1-4 \log 2<0$ we have $h^{\prime}(K)<0$ for $K \geqslant 1$. Hence $h$ is decreasing, so that $h(K) \leqslant 0$ for all $K \geqslant 1$.

2.24. THEOREM. For $n \geqslant 2, K \geqslant 1$, and $r \in[0,1]$,

$$
\varphi_{K, n}^{* 2}(r)+\varphi_{1 / K, n}^{2}\left(r^{\prime}\right) \leqslant 1
$$

and

$$
\varphi_{1 / K, n}^{* 2}(r)+\varphi_{K, n}^{2}\left(r^{\prime}\right) \geqslant 1,
$$

where $r^{\prime}=\sqrt{1-r^{2}}$. In each case there is equality for all $r$ when $n=2$ or $K=1$.

Proof. We prove only (2.25), since the proof of (2.26) is similar. By Lemma 2.3, $\tau_{n}\left(\sinh ^{2}\left(\rho^{\prime} / 2\right)\right) \geqslant \tau_{n}\left(\sinh ^{2}(\rho / 2)\right) / K$ for $f \in Q C_{K}\left(B^{n}\right), f(0)=0, \rho^{\prime}=\rho(f(x), f(y))$, and $\rho=\rho(x, y)$. Using the identity $\sinh ^{2} A=\left(\tanh ^{2} A\right) /\left(1-\tanh ^{2} A\right)$ and (1.1) we obtain

$$
\gamma_{n}\left(\left(1-\tanh ^{2}\left(\rho^{\prime} / 2\right)\right)^{-1 / 2}\right) \geqslant(1 / K) \gamma_{n}\left(\left(1-\tanh ^{2}(\rho / 2)\right)^{-1 / 2}\right) .
$$

Now set $y=0=f(y)$ and $|x|=r$. Since $\rho(x, y)=2 \operatorname{arctanh} r$ and $\rho(f(x), f(y))$ $=2 \operatorname{arctanh}|f(x)|$, we obtain

$$
\gamma_{n}\left(\left(1-|f(x)|^{2}\right)^{-1 / 2}\right) \geqslant(1 / K) \gamma_{n}\left(\left(1-r^{2}\right)^{-1 / 2}\right) .
$$

Solving (2.27) for $|f(x)|$ gives

$$
|f(x)|^{2} \leqslant 1-\varphi_{1 / K, n}^{2}\left(\sqrt{1-r^{2}}\right),
$$

from which (2.25) follows when we take the supremum over all such $f$.

When $n=2$, equality holds in (2.25) and (2.26) for each $K>0$ and $r \in[0,1]$. This fact follows immediately from (1.5) and 2.19.

2.28. Remark. In view of (1.4) and Sychev's conjecture (1.7) it is of interest to observe that $\varphi_{K, n}(r)$ is not majorized by $4^{1-\alpha} r^{\alpha}, \alpha=K^{1 /(1-n)}$, for $K>1$ and large $n$. This is true because by [AVV, (4.5)]

$$
r<\tanh (K \operatorname{arctanh} r) \leqslant \varphi_{K, n}(r)
$$


for all $n \geqslant 2, K>1$, and $r \in(0,1)$, while $4^{1-\alpha} r^{\alpha}$ tends to $r$ as $n$ tends to $\infty$. On the other hand, we do not know whether $\varphi_{K, n}(r)$ is majorized by $4^{1-1 / K} r^{1 / K}$ for $n \geqslant 3$ and $K>1$.

3. A sharp bound for $|f(x)-f(y)|$. Several well-known Hölder-continuity theorems already existing in the literature have been cited in the introduction. Although some of these results yield the best Hölder exponent $\alpha=K^{1 /(1-n)}$, it seems that very few give the correct limit behavior as $K \rightarrow 1$. We shall prove such a theorem, which is suggested by a problem of $\mathrm{T}$. Iwaniec.

For $x \in B^{n}$ and $M \in(0, \infty)$ we let $D(x, M)$ denote the non-Euclidean ball $\left\{z \in B^{n}: \rho(x, z)<M\right\}$. The Euclidean diameter of a set $E$ in $R^{n}$ will be denoted by $d(E)$. It follows easily from the well-known formula for $D(z, M)$ [Ah2, p. 86, (4), (5); Vu2, (2.4)] that

$$
d(D(z, M)) \leqslant d(D(0, M))=2 \tanh (M / 2) .
$$

Next, given $x, y \in B^{n}$ let $z \in B^{n}$ be such that $\rho(x, z)=\rho(y, z)=\rho(x, y) / 2$. Then

$$
|x-y| \leqslant d(D(z, \rho(x, y) / 2)) \leqslant 2 \tanh (\rho(x, y) / 4)
$$

for all $x, y \in B^{n}$.

Moreover, by [Ah2, (32), p. 27],

$$
|x-y|=[x, y] \tanh \frac{\rho(x, y)}{2} \geqslant(1-|x||y|) \tanh \frac{\rho(x, y)}{2}
$$

for all $x, y \in B^{n}$, where $[x, y]^{2}=|x|^{2}|y|^{2}-2 x \cdot y+1$.

In summary, we have

3.3. LEMMA. If $T \in G M\left(B^{n}\right)$ and $x, y \in B^{n}$, then

$$
(1-|T x||T y|) \tanh (\rho(x, y) / 2) \leqslant|T x-T y| \leqslant 2 \tanh (\rho(x, y) / 4) .
$$

The first bound is sharp if $T y=0$; the second is sharp if $T$ is a rotation fixing the origin and $x=-y$.

Proof. Because $T$ is an isometry in the Poincaré metric $\rho$ the result follows from (3.1) and (3.2).

The main result of this section, perhaps new even for $n=2$, is the following generalization of Lemma 3.3.

3.4. TheOREM. Let $f$ be a $K$-quasiregular mapping of $B^{n}$ into $B^{n}$. Then

$$
|f(x)-f(y)| \leqslant b_{K}\left(\tanh \frac{1}{2} \rho(x, y)\right)
$$

for all $x, y \in B^{n}$, where $b_{K}(s)=2 \varphi_{K, n}(s) /\left(1+\sqrt{1-\varphi_{K, n}^{2}(s)}\right)$. The result is sharp if $f$ is a rotation fixing the origin and $x=-y$.

ProOF. If we let $t^{\prime}=\frac{1}{2} \rho(f(x), f(y))$, it follows from (3.1) that

$$
|f(x)-f(y)| \leqslant 2 \tanh \frac{t^{\prime}}{2}=\frac{2 \tanh t^{\prime}}{1+\sqrt{1-\tanh ^{2} t^{\prime}}} .
$$

Since $\tanh t^{\prime} \leqslant \varphi_{K, n}\left(\tanh \frac{1}{2} \rho(x, y)\right)$ by [MRV, 3.1] (cf. [Vu2, 3.3]) and since the right side of (3.6) is an increasing function of $t^{\prime}$, we obtain (3.5). Since $\varphi_{1, n}(r) \equiv r$, the sharpness assertion follows from Lemma 3.3. 
3.7. COROLLARY. Under the hypotheses of Theorem 3.4,

$$
|f(x)-f(y)| \leqslant\left(2 \lambda_{n}\right)^{1-\alpha} \rho(x, y)^{\alpha}, \quad \alpha=K^{1 /(1-n)},
$$

and

$$
|f(x)-f(y)| \leqslant \varphi_{K, n}(a)+\varphi_{K, n}^{2}(a), \quad a=\tanh \frac{\rho(x, y)}{2},
$$

for all $x, y \in B^{n}$.

Proof. It follows from (3.1) and (1.6) (cf. [Vu2, 3.3(1)]) that

$$
\begin{aligned}
|f(x)-f(y)| & \leqslant 2 \tanh (\rho(f(x), f(y)) / 2) \\
& \leqslant 2 \varphi_{K, n}(\tanh (\rho(x, y) / 2)) \leqslant 2 \lambda_{n}^{1-\alpha}(\rho(x, y) / 2)^{\alpha}
\end{aligned}
$$

and (3.8) is proved. Formula (3.9) follows from (3.5) and the second part of the elementary inequality

$$
\frac{4(1+x)}{3+2 \sqrt{2}} \leqslant \frac{2}{1+\sqrt{1-x^{2}}} \leqslant 1+x
$$

for $0 \leqslant x \leqslant 1$.

A drawback of the sharp inequality (3.5) is its relatively complicated form. In order to derive a more practical alternative form we recall some facts about elliptic integrals. As in [LV, p. 60] we let

$$
\mu(r)=\frac{\pi \mathscr{K}\left(\sqrt{1-r^{2}}\right)}{2 \mathscr{K}(r)}, \quad \mathscr{K}(r)=\int_{0}^{1}\left[\left(1-x^{2}\right)\left(1-r^{2} x^{2}\right)\right]^{-1 / 2} d x,
$$

for each $r \in(0,1)$. We shall need the well-known inequality

$$
\log (1 / r)<\mu(r)<\log (4 / r), \quad 0<r<1
$$

(cf. [LV, p. 61]).

3.12. Lemma. For $n \geqslant 2, K \geqslant 1$, and $0<r<1$, the following inequalities hold:

$$
\frac{1+t^{2 K}}{\left(1+t^{K}\right)^{2}} \leqslant \frac{1}{1+\sqrt{1-\varphi_{K, n}^{2}(r)}} \leqslant \frac{1}{2}\left(1+\varphi_{K, n}(r)\right),
$$

where $t=((1-r) /(1+r))^{1 / 2}$. Moreover,

$$
\frac{1}{1+\sqrt{1-\varphi_{K, n}^{2}(r)}} \leqslant \frac{1+(t / 2)^{2 K}}{\left(1+(t / 2)^{K}\right)^{2}} .
$$

Proof. By [AVV, (4.5)],

$$
\frac{(1+r)^{K}-(1-r)^{K}}{(1+r)^{K}+(1-r)^{K}} \leqslant \varphi_{K, n}(r) \leqslant \tanh \left(\frac{K}{2} \mu\left(\frac{1-r}{1+r}\right)\right)
$$


for $K \geqslant 1$. The lower bound in (3.13) follows from the lower bound in (3.15). The upper bound in (3.13) is a consequence of the second inequality in (3.10). Finally, from the upper bounds in (3.15) and (3.11) we see that

$$
\varphi_{K, n}(r) \leqslant \tanh \left(K \log \frac{2}{t}\right)=\frac{1-(t / 2)^{2 K}}{1+(t / 2)^{2 K}},
$$

and (3.14) follows by an easy computation.

3.16. REMARK. For very small values of $r$, say for $r \in\left(0, r_{0}\right)$, where $\lambda_{n}^{1-\alpha} r_{0}^{\alpha}=1 / 2$ and $\alpha=K^{1 /(1-n)}$, one can use inequality (1.6) instead of [AVV, (4.5)] to prove an estimate for $\varphi_{K, n}$ that is slightly different from those in Lemma 3.12.

3.17. Proof of Theorem 1.10. Let $r \in(0,1)$ and $x, y \in \bar{B}^{n}(r)$. By (3.5), (1.6), (2.1), and (3.13),

$$
\begin{aligned}
|f(x)-f(y)| & \leqslant b_{K}\left(\tanh \frac{\rho(x, y)}{2}\right) \\
& \leqslant \frac{2 \lambda_{n}^{1-\alpha}(\tanh (\rho(x, y) / 2))^{\alpha}}{1+\sqrt{1-\varphi_{K, n}^{2}\left(2 r /\left(1+r^{2}\right)\right)}} \\
& \leqslant \frac{\lambda_{n}^{1-\alpha}\left(1+\varphi_{K, n}\left(2 r /\left(1+r^{2}\right)\right)\right)|x-y|^{\alpha}}{\left[|x-y|^{2}+\left(1-|x|^{2}\right)\left(1-|y|^{2}\right)\right]^{\alpha / 2}} .
\end{aligned}
$$

From these estimates and from [AVV, Theorem 4.4] it follows that we may choose

$$
\begin{aligned}
a(r) & =\left(1+\varphi_{K, n}\left(\frac{2 r}{1+r^{2}}\right)\right)\left(1-r^{2}\right)^{-\alpha} \\
& \leqslant\left(1+\tanh \left(\frac{K}{2} \mu\left(\left(\frac{1-r}{1+r}\right)^{2}\right)\right)\right)\left(1-r^{2}\right)^{-\alpha}
\end{aligned}
$$

in Theorem 1.10. Inequality (3.11) implies that $\lim _{r \rightarrow 0} a(r)=1$. Finally, $\lambda_{n}^{1-\alpha} \leqslant$ $2^{1-1 / K} K$ by [AVV, 4.14].

3.18. Remarks. (1) If we set $K=1$ and $n=2$ in (3.5) we find that the upper bound in Lemma 3.3 holds not just for $G M\left(B^{n}\right)$ but for all analytic functions $f$ : $B^{2} \rightarrow B^{2}$.

(2) C. Carathéodory [Ca, Satz 1] proved that

$$
|f(x)-f(y)| /|x-y| \leqslant 1
$$

for distinct points $x, y \in \bar{B}^{2}(\sqrt{2}-1)$ when $f: B^{2} \rightarrow B^{2}$ is an analytic function with $f(0)=0$. His result is closely related to Theorem 1.10 , although no normalization at 0 is required in 1.10 .

(3) For $f \in M\left(B^{n}\right)$ one may improve Theorem 1.10 in the following way. By conformal invariance of the hyperbolic metric, (2.2) yields the estimate

$$
|f(x)-f(y)| \leqslant \frac{1}{1-r^{2}}|x-y|
$$

for all $x, y \in \bar{B}^{n}(r), r \in(0,1)$. 
4. Distortion of the quasihyperbolic metric. Let $D, D^{\prime}$ be domains in $R^{n}, L \geqslant 1$, and $f: D \rightarrow D^{\prime}$ a homeomorphism such that

$$
|x-y| / L \leqslant|f(x)-f(y)| \leqslant L|x-y|
$$

for all $x, y \in D$. Then we say that $f$ is $L$-bi-Lipschitz. If (4.1) holds with $L=1$ then $f$ is called a Euclidean isometry.

4.2. Lemma. Let $D, D^{\prime}$ be proper subdomains of $R^{n}$ and let $f: D \rightarrow D^{\prime}$ be an L-bi-Lipschitz homeomorphism. Then

$$
L^{-2} k_{D}(a, b) \leqslant k_{D^{\prime}}(f(a), f(b)) \leqslant L^{2} k_{D}(a, b)
$$

for all $a, b \in D$, where $k_{D}$ is the quasihyperbolic metric defined in (1.11). In particular, $k_{D}$ is invariant under Euclidean isometries.

Proof. It is enough to prove the second inequality, since the first follows from application of the second to $f^{-1}$.

Suppose $\gamma$ is a rectifiable arc in $D$ joining $a$ to $b$. By Theorem 5.3 of [V, p. 12] we have

$$
\int_{f \circ \gamma} d\left(y, \partial D^{\prime}\right)^{-1}|d y| \leqslant L \int_{\gamma} d\left(f(x), \partial D^{\prime}\right)^{-1}|d x| .
$$

Choose $\zeta^{\prime} \in \partial D^{\prime}$ such that $d\left(f(x), \partial D^{\prime}\right)=\left|f(x)-\zeta^{\prime}\right|$. Then there exists a sequence $y_{m} \in D^{\prime}$ such that $\lim y_{m}=\zeta^{\prime}$. Let $x_{m}=f^{-1}\left(y_{m}\right)$. By choosing a subsequence, we may assume that $x_{m} \rightarrow \zeta \in \partial D$. Then

$$
\left|f(x)-y_{m}\right|=\left|f(x)-f\left(x_{m}\right)\right| \geqslant L^{-1}\left|x-x_{m}\right| \text {. }
$$

Hence letting $m$ tend to $\infty$ we get $d\left(f(x), \partial D^{\prime}\right)^{-1} \leqslant L d(x, \partial D)^{-1}$ for all $x \in \gamma$. The second inequality in (4.3) follows if we combine (4.4) and (4.5), then take the infimum over all $\gamma$ joining $a$ and $b$ in $D$.

We now define

$$
j_{D}(x, y)=\log (1+|x-y| / \min \{d(x), d(y)\}) \text {, }
$$

where $d(z)=d(z, \partial D)$ for $z \in D$ (cf. [GO; Vu2, (2.26)]).

4.6. COROLlaRY. Under the hypotheses of Lemma 4.2, we have

$$
L^{-2} j_{D}(x, y) \leqslant j_{D^{\prime}}(f(x), f(y)) \leqslant L_{j_{D}}^{2}(x, y)
$$

for all $x, y \in D$.

Proof. As in Lemma 4.2, it is enough to prove the second inequality. We may assume that $d\left(f(x), \partial D^{\prime}\right) \leqslant d\left(f(y), \partial D^{\prime}\right)$. Then

$$
\begin{aligned}
j_{D^{\prime}}(f(x), f(y)) & =\log \left(1+|f(x)-f(y)| / d\left(f(x), \partial D^{\prime}\right)\right) \\
& \leqslant \log \left(1+L^{2}|x-y| / d(x, \partial D)\right) \\
& \leqslant L^{2} \log (1+|x-y| / d(x, \partial D)) \leqslant L^{2} j_{D}(x, y) .
\end{aligned}
$$

Here we have used the proof of Lemma 4.2 and Bernoulli's inequality $t \log (1+x) \geqslant$ $\log (1+t x)$ for $x \geqslant 0$ and $t \geqslant 1$ (cf. [Vu1, (2.33)]).

The next lemma generalizes [GO, Lemma 2]. 
4.7. Lemma. For each $K \geqslant 1$ there exists a constant $a_{1} \in\left(\left(\frac{1}{2}(\sqrt{3}-\sqrt{2})\right)^{8 K}\right.$, $\left.2(\sqrt{3}-\sqrt{2})^{8 K}\right)$ with the following property. If $D, D^{\prime}$ are proper subdomains of $R^{n}$, $n \geqslant 2$, and if $f: D \rightarrow D^{\prime}$ is $K$-quasiconformal, then

$$
|f(x)-f(y)| / d\left(f(x), \partial D^{\prime}\right) \leqslant 1 / 2
$$

and

$$
\frac{|f(x)-f(y)|}{d\left(f(x), \partial D^{\prime}\right)} \leqslant \frac{1}{2} \varphi_{K, n}^{*}\left(\frac{|x-y|}{a_{1} d(x, \partial D)}\right) \leqslant \frac{K}{2^{1 / K} a_{1}}\left(\frac{|x-y|}{d(x, \partial D)}\right)^{\alpha}
$$

for $y \in B^{n}\left(x, a_{1} d(x, \partial D)\right)$.

Proof. The second assertion follows easily from the first one and from definitions (1.3), (1.4), and (1.6). Hence it suffices to prove the first assertion. Since only the maximal inscribed ball $B^{n}(x, d(x, \partial D))$ will matter in the proof, we may assume that $D=B^{n}$ and $x=0$. Let

$$
r_{D^{\prime}}(u, v)=\frac{|u-v|}{\min \left\{d\left(u, \partial D^{\prime}\right), d\left(v, \partial D^{\prime}\right)\right\}}
$$

for $u, v \in D^{\prime}$. Then by [Vu3, Theorem 4.5],

$$
\lambda_{D^{\prime}}(f(x), f(y)) \leqslant 4 \tau\left(r_{D^{\prime}}(f(x), f(y))\right),
$$

where $\lambda_{D^{\prime}}$ represents a conformal invariant studied in [Vu2]. By (4.8), [Vu2, Lemma $3.1(2)],(2.6)$, and (2.2), we obtain

$$
r_{D^{\prime}}(f(0), f(y)) \leqslant \tau^{-1}\left(\frac{1}{8 K} \tau\left(\frac{|y|^{2}}{1-|y|^{2}}\right)\right) .
$$

Next, if we require that

$$
\tau^{-1}\left(\frac{1}{8 K} \tau\left(\frac{|y|^{2}}{1-|y|^{2}}\right)\right) \leqslant \frac{1}{2}
$$

and solve for $|y|$, we obtain the upper bound

$$
|y|^{2} \leqslant \tau^{-1}\left(8 K \tau\left(\frac{1}{2}\right)\right) /\left(1+\tau^{-1}\left(8 K \tau\left(\frac{1}{2}\right)\right)\right) \text {. }
$$

Thus we may choose $a_{1}$ as $\left[\tau^{-1}\left(8 K \tau\left(\frac{1}{2}\right)\right) /\left(1+\tau^{-1}\left(8 K \tau\left(\frac{1}{2}\right)\right)\right)\right]^{1 / 2}$ in the theorem or, in light of (1.1) and (1.2),

$$
a_{1}=\sqrt{1-\varphi_{8 K, n}^{2}(\sqrt{2 / 3})} .
$$

Finally, from (3.13) and (3.14), with $r=\sqrt{2 / 3}$ and with $K$ replaced by $8 K$, we obtain

$$
\frac{2(t / 2)^{8 K}}{1+(t / 2)^{16 K}} \leqslant a_{1}=\sqrt{1-\varphi_{8 K, n}^{2}(r)} \leqslant \frac{2 t^{8 K}}{1+t^{16 K}},
$$

with $t=\sqrt{3}-\sqrt{2}$. Thus

$$
\left(\frac{1}{2}(\sqrt{3}-\sqrt{2})\right)^{8 K}<a_{1}<2(\sqrt{3}-\sqrt{2})^{8 K} .
$$


4.10. LEMMA. If $D$ is a proper subdomain of $R^{n}$ then

$$
k_{D}(x, y) \geqslant j_{D}(x, y)
$$

for all $x, y \in D$ and

$$
k_{D}(x, y) \leqslant \log (1+|x-y| /(d(x)-|x-y|))
$$

for $|x-y|<d(x)$, where $d(x)=d(x, \partial D)$.

Proof. Inequality (4.11) is [GP, (2.2)], and (4.12) is [Vu1, Lemma 2.11].

4.13. Proof of Theorem 1.12. In [GO, Theorem 3] this result was proved with the constant $c=4\left(4 \lambda_{n}^{2}\right)^{1 / \alpha}$, which tends to $\infty$ as $n$ tends to $\infty$ [An]. We need to show that we can replace this with a universal constant $c$ that does not depend on $n$. It suffices to prove the first inequality in Theorem 1.12 since

$$
\max \left\{k_{D}(x, y), k_{D}(x, y)^{\alpha}\right\}=k_{D}(x, y)^{\alpha} \leqslant k_{D}(x, y)^{1 / K}
$$

for $k_{D}(x, y) \leqslant 1$, and

$$
\max \left\{k_{D}(x, y), k_{D}(x, y)^{\alpha}\right\}=k_{D}(x, y)=\max \left\{k_{D}(x, y), k_{D}(x, y)^{1 / K}\right\}
$$

for $k_{D}(x, y) \geqslant 1$.

Suppose first that

$$
|x-y| \leqslant a_{1} d(x, \partial D),
$$

where $a_{1}$ is as in Lemma 4.7. Then by Lemma 4.7,

$$
|f(x)-f(y)| / d\left(f(x), \partial D^{\prime}\right) \leqslant 1 / 2
$$

and hence by (4.12)

$$
\begin{aligned}
k_{D^{\prime}}(f(x), f(y)) & \leqslant \log \left(1+\frac{|f(x)-f(y)|}{d\left(f(x), \partial D^{\prime}\right)-|f(x)-f(y)|}\right) \\
& \leqslant \frac{2|f(x)-f(y)|}{d\left(f(x), \partial D^{\prime}\right)} \leqslant 1
\end{aligned}
$$

since $\log (1+t) \leqslant t$ for $t \geqslant 0$.

On the other hand, since $|x-y| / d(x, \partial D) \leqslant a_{1}$ and since $(1 / t) \log (1+t)$ is decreasing for $t>0$ we see by (4.11) that

$$
k_{D}(x, y) \geqslant \log \left(1+\frac{|x-y|}{d(x, \partial D)}\right) \geqslant \frac{\log \left(1+a_{1}\right)}{a_{1}} \frac{|x-y|}{d(x, \partial D)} .
$$

Then by Lemma 4.7 we obtain

$$
|f(x)-f(y)| \leqslant \frac{1}{2} d\left(f(x), \partial D^{\prime}\right) \varphi_{K, n}^{*}\left(|x-y| /\left(a_{1} d(x, \partial D)\right)\right) .
$$

Thus by (4.16), (4.18), (1.4), (1.6), and (4.17) we have

$$
\begin{aligned}
k_{D^{\prime}}(f(x), f(y)) & \leqslant \varphi_{K, n}^{*}\left(\frac{|x-y|}{a_{1} d(x, \partial D)}\right) \leqslant 2^{1-1 / K} K\left(\frac{|x-y|}{a_{1} d(x, \partial D)}\right)^{\alpha} \\
& \leqslant 2^{1-1 / K} K\left(\frac{k_{D}(x, y)}{\log \left(1+a_{1}\right)}\right)^{\alpha} .
\end{aligned}
$$


Next suppose that

$$
|x-y|>a_{1} d(x, \partial D)
$$

and choose $z_{1}, \ldots, z_{m+1}$ on a quasihyperbolic geodesic in $D$ joining $x$ and $y$ [GO] so that $z_{1}=x, z_{m+1}=y$, and

$$
\left|z_{l}-z_{l+1}\right| / d\left(z_{l}, \partial D\right)=a_{1}, \quad\left|z_{m}-z_{m+1}\right| / d\left(z_{m}, \partial D\right) \leqslant a_{1}
$$

for $l=1, \ldots, m-1$. Then by (4.11)

$$
k_{D}(x, y) \geqslant \sum_{l=1}^{m-1} k_{D}\left(z_{l}, z_{l+1}\right) \geqslant(m-1) \log \left(1+a_{1}\right) \text {. }
$$

Hence

$$
m \leqslant 1+k_{D}(x, y) / \log \left(1+a_{1}\right) .
$$

Then by (4.12), the definition of the number $a_{1}$, and (4.22),

$$
\begin{aligned}
k_{D^{\prime}}(f(x), f(y)) & \leqslant \sum_{l=1}^{m} k_{D^{\prime}}\left(f\left(z_{l}\right), f\left(z_{l+1}\right)\right) \\
& \leqslant \sum_{l=1}^{m} \log \left(1+\frac{\left|f\left(z_{l}\right)-f\left(z_{l+1}\right)\right|}{d\left(f\left(z_{l}\right), \partial D^{\prime}\right)-\left|f\left(z_{l}\right)-f\left(z_{l+1}\right)\right|}\right) \\
& \leqslant\left(1+\frac{k_{D}(x, y)}{\log \left(1+a_{1}\right)}\right) \log 2 .
\end{aligned}
$$

Since $k_{D}(x, y) \geqslant j_{D}(x, y) \geqslant \log \left(1+a_{1}\right),(4.23)$ now yields

$$
k_{D^{\prime}}(f(x), f(y)) \leqslant 2(\log 2) k_{D}(x, y) / \log \left(1+a_{1}\right),
$$

provided (4.20) holds.

Finally by (4.19) and (4.24) we obtain, for all $x, y \in D$,

$$
\begin{aligned}
k_{D^{\prime}}(f(x), f(y)) & \leqslant \max \left\{b_{1} k_{D}(x, y)^{\alpha}, b_{2} k_{D}(x, y)\right\} \\
& \leqslant \max \left\{b_{1}, b_{2}\right\} \max \left\{k_{D}(x, y)^{\alpha}, k_{D}(x, y)\right\},
\end{aligned}
$$

where $b_{1}=2^{1-1 / K} K\left(\log \left(1+a_{1}\right)\right)^{-\alpha}$ and $b_{2}=(2 \log 2) / \log \left(1+a_{1}\right)$. From Lemma 4.7 it follows easily that

$$
\max \left\{b_{1}, b_{2}\right\} \leqslant 2 K / \log \left(1+a_{1}\right) .
$$

Therefore, by (4.25), we may choose $c(K)=2 K / \log \left(1+a_{1}\right)$ in Theorem 1.12 .

Next, we define

$$
\bar{c}(K)=\inf \{c(K): \text { Theorem } 1.12 \text { holds with } c(K)\} .
$$

We show that $\bar{c}(K) \rightarrow \infty$ as $K \rightarrow \infty$ and provide quantitative lower and upper bounds. To this end the following two lemmas are needed.

4.27. Lemma. Let $G=R^{n} \backslash\{0\}$, let $x, y \in G$, and let $\varphi$ be the angle determined by the segments $[0, x]$ and $[0, y], \varphi \in[0, \pi]$. Then

$$
k_{G}(x, y)=\sqrt{\varphi^{2}+\log ^{2}(|x| /|y|)} .
$$

Proof. This formula is developed in [MO, §2]. 
Now let $\lambda(K)=\left(\mu^{-1}(\pi / 2 K) / \mu^{-1}(\pi K / 2)\right)^{2}$ denote the well-known distortion coefficient of Lehto, Virtanen, and Väisälä [LVV] (cf. [LV, pp. 81, 82, 106-108]). In particular, $\lambda(1)=1$ and $\lambda(K)$ tends to $\infty$ as $K$ tends to $\infty$.

4.28. Lemma. There exists a $K$-quasiconformal mapping $f: R^{n} \rightarrow R^{n}$ such that $f(0)=0, f(\infty)=\infty, f\left(e_{1}\right)=e_{1}$, and $f\left(-e_{1}\right)=-\lambda\left(K^{1 /(n-1)}\right) e_{1}$.

PRoof. This follows by rotation of the two-dimensional extremal quasiconformal mapping of [LVV] (cf. [AVV, Theorem 1.14]).

4.29. THEOREM. The constant $\bar{c}(K)$ in (4.26) satisfies

$$
\left[1+\pi^{-2} \log ^{2} \lambda(K)\right]^{1 / 2} \leqslant \bar{c}(K) \leqslant 2 K\left[1+(2(\sqrt{3}+\sqrt{2}))^{8 K}\right] .
$$

Proof. For the lower bound fix $f$ as in Lemma 4.28. Then with $D=R^{n} \backslash\{0\}$, we have by Lemma 4.27 the relations

$$
\ell_{D}\left(e_{1},-e_{1}\right)=\pi
$$

and

$$
\begin{aligned}
k_{D}\left(f\left(e_{1}\right), f\left(-e_{1}\right)\right) & =k_{D}\left(e_{1},-\lambda\left(K^{1 /(n-1)}\right) e_{1}\right) \\
& =\left[\pi^{2}+\log ^{2} \lambda\left(K^{1 /(n-1)}\right)\right]^{1 / 2} .
\end{aligned}
$$

Thus by the definition of $\bar{c}(K)$ we have

$$
\left[\pi^{2}+\log ^{2} \lambda\left(K^{1 /(n-1)}\right)\right]^{1 / 2} \leqslant \pi \bar{c}(K) .
$$

Choosing $n=2$ yields the desired lower estimate.

Finally, by the choice of $c(K)$ in the proof of Theorem 1.12 , the estimate $\log \left(1+a_{1}\right)>a_{1} /\left(1+a_{1}\right)$, and Lemma 4.7, we have

$$
\bar{c}(K) \leqslant \frac{2 K}{\log \left(1+a_{1}\right)}<2 K\left(1+1 / a_{1}\right)<2 K\left[1+(2(\sqrt{3}+\sqrt{2}))^{8 K}\right],
$$

and the upper estimate follows.

We next obtain dimension-free versions of distortion results due to Gehring and Osgood [GO, Lemmas 2 and 3].

4.31. LeMmA. If $f$ is a $K$-quasiconformal mapping of $\bar{R}^{n}$ with $f(\infty)=\infty$, then

$$
\frac{|f(x)-f(y)|}{|f(x)-f(z)|}+1 \leqslant B\left(\left(\frac{|x-y|}{|x-z|}\right)^{K}+1\right),
$$

where $B=\left(1 / a_{1}\right) \max \left\{2, \frac{1}{2}(\min \{2, K\})^{K}\right\}$.

Proof. Let $D=R^{n} \backslash\{y\}, \quad D^{\prime}=R^{n} \backslash\{f(y)\}$. Then $|f(x)-f(y)|=$ $d\left(f(x), \partial D^{\prime}\right),|x-y|=d(x, \partial D)$. We may assume that

$$
|f(x)-f(z)| /|f(x)-f(y)| \leqslant 2 / B,
$$


since otherwise the result holds trivially. Then $|f(x)-f(z)| \leqslant a_{1} d\left(f^{\prime}(x), \partial D^{\prime}\right)$. So by (2.9) and Lemma 4.7 applied to $f^{-1}$ we get

$$
\begin{aligned}
\frac{|x-z|}{|x-y|} & \leqslant \frac{1}{2} \varphi_{K, n}^{*}\left(\frac{|f(x)-f(z)|}{a_{1}|f(x)-f(y)|}\right) \\
& \leqslant 2^{-1 / K} \min \{2, K\}\left(\frac{|f(x)-f(z)|}{a_{1}|f(x)-f(y)|}\right)^{1 / K},
\end{aligned}
$$

or

$$
\frac{|f(x)-f(y)|}{|f(x)-f(z)|} \leqslant \frac{(\min \{2, K\})^{K}}{2 a_{1}}\left(\frac{|x-y|}{|x-z|}\right)^{K} \leqslant B\left(\frac{|x-y|}{|x-z|}\right)^{K},
$$

and the result follows.

4.32. COROllaRY. Theorem 4 and Corollary 3 in [GO] hold with constants that do not depend on $n$.

Proof. In [GO, Theorem 4] we can choose $c=2 / \alpha=2 \beta \leqslant 2 K$ (as indicated in [GO]) and $d=\log B \leqslant \log \left(\max \left\{2,2(\min \{2, K\})^{K}\right\} / a_{1}\right)$. In [GO, Corollary 3] we may choose $c_{1}=c(K), c_{2}=2 \beta \leqslant 2 K, d_{2}=\log B$. Then the proof follows as in [GO].

\section{REFERENCES}

[Ah1] L. V. Ahlfors, On quasiconformal mappings, J. Analyse Math. 3 (1954), 1-58 and 207-208.

[Ah2] Möbius transformations in several variables, Univ. of Minnesota Lecture Notes, Minneapolis, 1981.

[An] G. D. Anderson, Dependence on dimension of a constant related to the Grötzsch ring, Proc. Amer. Math. Soc. 61 (1976), 77-80.

[AVV] G. D. Anderson, M. K. Vamanamurthy, and M. Vuorinen, Dimension-free quasiconformal distortion in n-space, Trans. Amer. Math. Soc. 297 (1986), 687-706.

[B] A. F. Beardon, The geometry of discrete groups, Graduate Texts in Math., vol. 91, Springer-Verlag, Berlin, Heidelberg and New York, 1983.

[C] E. D. Callender, Hölder continuity of n-dimensional quasiconformal mappings, Pacific J. Math. 10 (1960), 499-515.

[Ca] C. Carathéodory, Üher das Maximum des absoluten Betrages des Differenzenquotienten für unimodular beschränkte Funktionen, Math. Z. 47 (1942), 468-488.

[G] F. W. Gehring, Rings and quasiconformal mappings in space, Trans. Amer. Math. Soc. 103 (1962), 353-393.

[GO] F. W. Gehring and B. G. Osgood, Uniform domains and the quasi-hyperbolic metric, J. Analyse Math. 36 (1979), 50-74.

[GP] F. W. Gehring and B. P. Palka, Quasiconformally homogeneous domains, J. Analyse Math. 30 (1976), 172-199.

[HP] J. Hersch and A. Pfluger, Généralisation du lemme de Schwarz et du principe de la mesure harmonique pour les fonctions pseudo-analytiques, C. R. Acad. Sci. Paris 234 (1952), 43-45.

[I] T. Iwaniec, Some aspects of partial differential equations and quasiregular mappings, Proc. 1982 Internat. Congr. Math. (Warsaw, Poland), Vol. 2, PWN, Polish Scientific Publishers, Warzawa, 1984, 1193-1208.

[LV] O. Lehto and K. I. Virtanen, Quasiconformal mappings in the plane, 2nd ed., Grundlehren der math. Wissenschaften, vol. 126, Springer-Verlag, New York, Heidelberg and Berlin, 1973.

[LVV] O. Lehto, K. I. Virtanen, and J. Väisälä, Contributions to the distortion theory of quasiconformal mappings, Ann. Acad. Sci. Fenn. Ser. AI 273 (1959), 1-14. 
[MO] G. J. Martin and B. G. Osgood, The quasihyperbolic metric and associated estimates on the hyperbolic metric, J. Analyse Math. 47 (1986), 37-53.

[MRV] O. Martio, S. Rickman, and J. Väisälä, Distortion and singularities of quasiregular mappings, Ann. Acad. Sci. Fenn. Ser. AI 465 (1970), 1-13.

[R1] Yu. G. Reshetnyak, A sufficient condition for Hölder continuity of a mapping, Soviet Math. Dokl. 1 (1960), 76-78.

[R2] __ Bounds on moduli of continuity' for certain mappings, Siberian Math. J. 7 (1966), 879-886.

[R3] _. Spatial mappings with bounded distortion, "Nauka" Sibirsk. Otdel., Novosibirsk, 1982.

(Russian)

[Sh] B. V. Shabat, On the theory of quasiconformal mappings in space, Soviet Math. 1 (1960), 730-733.

[Sy] A. V. Sychev, Moduli and quasiconformal mappings in space, "Nauka" Sibirsk. Otdel., Novosibirsk, 1983. (Russian)

[V] J. Väisälä, Lectures on n-dimensional quasiconformal mappings, Lecture Notes in Math., vol. 229, Springer-Verlag, Berlin, Heidelberg and New York, 1971.

[Vu1] M. Vuorinen, Capacity densities and angular limits of quasiregular mappings, Trans. Amer. Math. Soc. 263 (1981), 343-354.

[Vu2] __, Conformal invariants and quasiregular mappings, J. Analyse Math. 45 (1985), 69-115.

[Vu3] _ On Teichmüller's modulus problem in $R^{n}$ (in preparation).

[W] Chuan-Fang Wang, On the precision of Mori's theorem in Q-mapping, Science Record 4 (1960), 329-333.

Department of Mathematics, Michigan State University, East Lansing, Michigan 48824

Department of Mathematics, University of Auckland, Auckland, New Zealand

Department of Mathematics, University of Helsinki, Helsinki, Finland 